\title{
Factors Controlling Rapid Stratocumulus Cloud Thinning
}

\author{
J. J. VAN DER DUSSEN AND S. R. DE ROODE \\ Delft University of Technology, Delft, Netherlands \\ A. P. SIEBESMA \\ Royal Netherlands Meteorological Institute, De Bilt, and Delft University of Technology, Delft, Netherlands
}

(Manuscript received 15 April 2013, in final form 4 October 2013)

\begin{abstract}
The relationship between the inversion stability and the liquid water path (LWP) tendency of a vertically well-mixed, adiabatic stratocumulus cloud layer is investigated in this study through the analysis of the budget equation for the LWP. The LWP budget is mainly determined by the turbulent fluxes of heat and moisture at the top and the base of the cloud layer, as well as by the source terms due to radiation and precipitation. Through substitution of the inversion stability parameter $\kappa$ into the budget equation, it immediately follows that the LWP tendency will become negative for increasing values of $\kappa$ due to the entrainment of increasingly dry air. Large $\kappa$ values are therefore associated with strong cloud thinning. Using the steady-state solution for the LWP, an equilibrium value $\kappa_{\text {eq }}$ is formulated, beyond which the stratocumulus cloud will thin. The Second Dynamics and Chemistry of Marine Stratocumulus field study (DYCOMS-II) is used to illustrate that, depending mainly on the magnitude of the moisture flux at cloud base, stratocumulus clouds can persist well within the buoyancy reversal regime.
\end{abstract}

\section{Introduction}

Buoyancy reversal at the top of a stratocumulus layer has often been suggested as a major stratocumulus cloud-dissolving mechanism. Lilly (1968) hypothesized that under certain inversion conditions, parcels that are entrained from above the inversion can become negatively buoyant by mixing with saturated air inside the cloud layer. As these parcels sink, turbulence kinetic energy is generated such that additional entrainment is promoted. Such runaway entrainment would rapidly warm and dry the cloud layer, leading to its breakup.

Randall (1980) and Deardorff (1980) formulated a criterion for this process to occur by determining the minimum buoyancy of entrained parcels. This criterion can be expressed in terms of an inversion stability parameter $\kappa$, which is a function of the ratio of the inversion jumps of total specific humidity $\Delta q_{t}$ and liquid water potential temperature $\Delta \theta_{l}$, as follows:

Corresponding author address: Johan van der Dussen, Civil Engineering and Geosciences, Delft University of Technology, Stevinweg 1, Delft 2628 CN, Netherlands.

E-mail: j.j.vanderdussen@tudelft.nl

$$
\kappa \equiv 1+\frac{c_{p}}{L_{v}} \frac{\Delta \theta_{l}}{\Delta q_{t}}
$$

Here, $c_{p}$ is the specific heat of air and $L_{v}$ is the latent heat of vaporization of water. Siems et al. (1990) showed that using Eq. (1), the Randall and Deardorff criterion can conveniently be written as $\kappa>\kappa_{\mathrm{BR}}$, where $\kappa_{\mathrm{BR}}=0.23$. Similar criteria have been suggested by, among others, MacVean and Mason (1990), Siems et al. (1990), and Duynkerke (1993) [for an overview, see Yamaguchi and Randall (2008)].

However, Kuo and Schubert (1988) found that most of the available stratocumulus observations lie within the buoyancy reversal regime. Siems et al. (1990) furthermore performed laboratory experiments from which they concluded that a positive entrainment feedback due to buoyancy reversal does not occur under realistic stratocumulus conditions. Similar conclusions are drawn from recent high-resolution large-eddy simulation (LES) results by Yamaguchi and Randall (2008), who find that spontaneous entrainment as a result of evaporative cooling indeed exists, but the effect is weak and does not lead to runaway entrainment. Mellado et al. (2009) furthermore conclude from linear stability and numerical 
analyses that evaporative cooling of entrained parcels does enhance the turbulence generation slightly below the inversion, but the entrainment velocity is not affected.

Nevertheless, the LES results of Moeng (2000) and more recently of Lock (2009) strongly suggest that cloud cover in stably stratified boundary layers tends to decrease rapidly beyond a certain critical value for $\kappa$. Similarly, Noda et al. (2013) show from LESs of transient stratocumulus-topped boundary layers that for larger values of $\kappa$, the LWP tendency is more negative and the cloud layer tends to break up earlier.

The $\kappa$ dependency of cloud cover is particularly interesting in connection with climate perturbation studies. The value of $\kappa$ will typically increase in climate-warming scenarios, as $\Delta \theta_{l}$ remains approximately constant, while the humidity jump increases as a result of ClausiusClapeyron scaling (Bretherton et al. 2013; Bretherton and Blossey 2013, manuscript submitted to $J$. $A d v$. Model. Earth Syst.). A thorough understanding of how this will affect the low cloud cover is important for determining the magnitude of the cloud-climate feedback (Stephens 2005).

Stratocumulus-to-cumulus transitions that are often observed over subtropical oceans provide further motivation for the research presented in this article. As stratocumulus cloud fields are advected toward the equator, $\Delta q_{t}$ typically increases due to the deepening of the boundary layer in combination with the negative humidity gradient in the free atmosphere, and the increase of the surface saturation specific humidity as the sea surface temperature increases. The temperature jump typically changes less rapidly, as the sea surface temperature increase counteracts the stabilizing effect of boundary layer deepening. The increase of $\Delta q_{t}$ therefore dominates the change in $\kappa$, causing it to increase. Eventually the stratocumulus cloud breaks up and a transition to cumulus clouds is observed.

Four stratocumulus transitions have been simulated as a model intercomparison of the combined Global Atmospheric System Studies (GASS) and European Union Cloud Intercomparison, Process Study and Evaluation (EUCLIPSE) projects. These cases mainly differ in the magnitude of the initial temperature and humidity jumps. For a detailed description of the three composite cases, see Sandu and Stevens (2011). The setup of the transition based on the Atlantic Stratocumulus Transition Experiment (ASTEX; Albrecht et al. 1995) is described by Van der Dussen et al. (2013). The 2-hourly averaged values of the cloud fraction as a function of $\kappa$ for these four cases, obtained using the Dutch Atmospheric LES (DALES) model, are shown in Fig. 1. Sandu and Stevens (2011) presented in a similar figure the results of the composite transition cases obtained

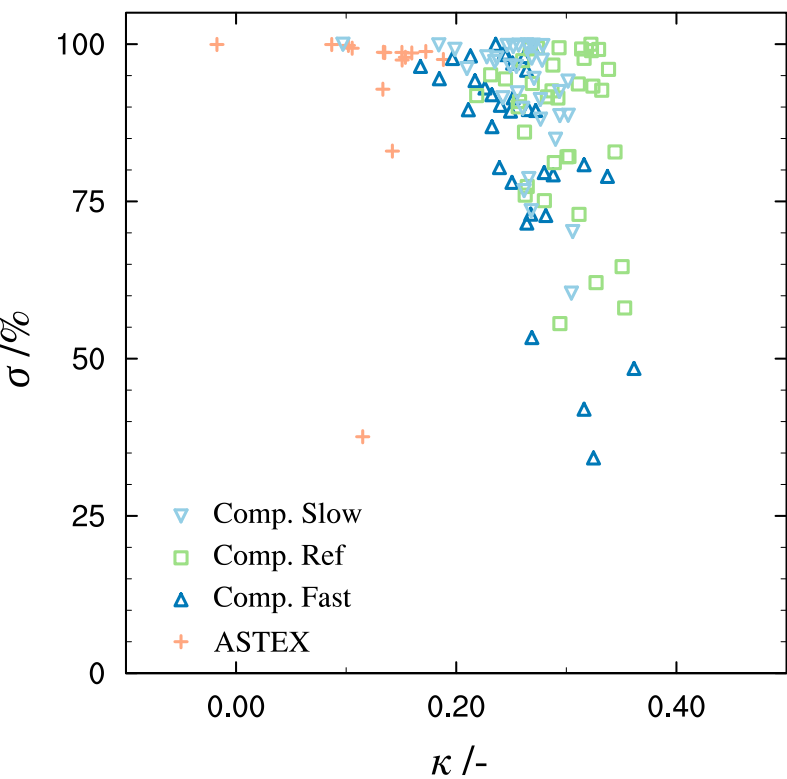

FIG. 1. The 2-hourly averaged cloud cover as a function of $\kappa$ for the four GASS-EUCLIPSE model intercomparison cases, as indicated by the legend. Simulations where performed using the DALES model (Heus et al. 2010).

using the University of California, Los Angeles (UCLA) LES model. These results also indicate that the cloud fraction $\sigma$ decreases rapidly beyond some critical $\kappa$.

For the ASTEX transition, this critical $\kappa$ value is clearly lower than for the composite transition cases. This apparent lack of a universal critical value for $\kappa$ has also been found by Xiao et al. (2011), who concluded that decoupling of the boundary layer causes stratocumulus breakup to occur at lower $\kappa$ values. The study thus shows that the critical value for $\kappa$ depends on the moisture supply at the stratocumulus cloud base. These examples cannot be sufficiently explained by the existing $\kappa$ criteria that are based on buoyancy reversal argumentation.

In the following section, we will derive an equation for the tendency of the liquid water path (LWP) of adiabatic stratocumulus cloud layers. This equation is then rewritten in terms of $\kappa$, which shows that for sufficiently large values of $\kappa$ the cloud-thinning tendency due to entrainment drying and warming becomes so large that it cannot be compensated anymore by cloudbuilding processes. In section 3 , a simple entrainment relation is assumed that allows for the derivation of an equilibrium value of $\kappa$ for which the LWP is constant in time. The results are furthermore linked to the Klein and Hartmann (1993) relation, which describes the cloud cover as a function of the bulk stability of the boundary layer. The final section contains a short summary of the conclusions. 


\section{Theory}

Randall (1984) derived an equation for the tendency of $q_{l}^{t}$, the liquid water specific humidity at the top of a well-mixed stratocumulus-topped boundary layer, in order to investigate the effect of entrainment on the cloud layer. For such boundary layers, $q_{l}^{t}$ is a function of $q_{t}$ and $\theta_{l}$ in the cloud layer, as well as of the inversion height $z_{i}$, such that the following can be written:

$$
\frac{\partial q_{l}^{t}}{\partial t}=\frac{\partial q_{l}^{t}}{\partial q_{t}} \frac{\partial q_{t}}{\partial t}+\frac{\partial q_{l}^{t}}{\partial \theta_{l}} \frac{\partial \theta_{l}}{\partial t}+\frac{\partial q_{l}^{t}}{\partial z_{i}} \frac{\partial z_{i}}{\partial t}
$$

Note that the partial derivatives imply that all but the variable of interest are kept constant.

Using the trapezoidal rule, $q_{l}^{t}$ can be related to the LWP, giving

$$
\mathrm{LWP}=\int_{z=0}^{\infty} \rho q_{l} d z \approx \frac{1}{2} \rho h q_{l}^{t} .
$$

Here, $\rho$ is the total density of air and $h$ is the thickness of the cloud layer. This relation assumes that $q_{l}$ varies approximately linearly with height in the cloud layer,

$$
\frac{\partial q_{l}}{\partial z}=-\Gamma_{q_{l}}
$$

in which $\Gamma_{q_{l}}$ is the lapse rate of the liquid water specific humidity. We assume that the cloud layer is vertically well mixed as a result of the destabilizing effect of longwave radiative cooling at cloud top and accordingly the $q_{l}$ lapse rate is adiabatic. Following the conceptual model for stratocumulus-topped boundary layers of Park et al. (2004) and Wood and Bretherton (2004), the stratocumulus cloud layer is allowed to have a different $\theta_{l}$ and $q_{t}$ than the subcloud layer. Both layers are then connected by a conditionally unstable layer in which cumuli are responsible for the transport of moisture to the stratocumulus layer (e.g., Wang and Lenschow 1995). In other words, the stratocumulus layer is well mixed even though the boundary layer as a whole is possibly decoupled.

Integration of Eq. (4) shows that $q_{l}^{t}=-\Gamma_{q_{l}} h$, such that the LWP tendency can be written as follows:

$$
\frac{\partial \mathrm{LWP}}{\partial t}=-\frac{1}{2} \frac{\partial}{\partial t}\left(\rho \Gamma_{q_{l}} h^{2}\right) \approx-\rho h \frac{\partial}{\partial t}\left(\Gamma_{q_{l}} h\right)=\rho h \frac{\partial q_{l}^{t}}{\partial t} .
$$

This equation shows that the LWP tendency is linearly related to the tendency of $q_{l}^{t}$ in Eq. (2).

The following expressions for the partial derivatives of $q_{l}^{t}$ in Eq. (2) are derived in the appendix:

$$
\begin{aligned}
& \frac{\partial q_{l}^{t}}{\partial q_{t}}=\eta, \\
& \frac{\partial q_{l}^{t}}{\partial \theta_{l}}=-\Pi \gamma \eta,
\end{aligned}
$$

where the variable

$$
\eta=\left(1+\frac{L_{v} \gamma}{c_{p}}\right)^{-1}
$$

accounts for the latent heat release (uptake) associated with the condensation (evaporation) of liquid water. The value of $\eta$ depends mainly on temperature. For subtropical stratocumulus clouds, $\eta \approx 0.4$. Furthermore, $\Pi$ is the Exner function and $\gamma=\partial q_{s} / \partial T$ is the change of the saturation specific humidity $q_{s}$ with temperature $T$ as described by the Clausius-Clapeyron relation.

The entrainment rate and the large-scale vertical velocity $\bar{w}$ together determine the rate of change of the inversion height with time:

$$
\frac{\partial z_{i}}{\partial t}=w_{e}+\bar{w}\left(z_{i}\right) .
$$

Through substitution of Eqs. (5), (6), and (8), Eq. (2) becomes

$$
\frac{1}{\rho h} \frac{\partial \mathrm{LWP}}{\partial t}=\eta \frac{\partial q_{t}}{\partial t}-\Pi \gamma \eta \frac{\partial \theta_{l}}{\partial t}-\Gamma_{q_{l}}\left[w_{e}+\bar{w}\left(z_{i}\right)\right] .
$$

The tendencies of $q_{t}$ and $\theta_{l}$ in this equation are governed by their respective Reynolds-averaged budget equations:

$$
\begin{aligned}
& \frac{\partial q_{t}}{\partial t}=-\frac{\partial \overline{w^{\prime} q_{t}^{\prime}}}{\partial z}-\frac{\partial P}{\partial z} \\
& \frac{\partial \theta_{l}}{\partial t}=-\frac{\partial \overline{w^{\prime} \theta_{l}^{\prime}}}{\partial z}-\frac{1}{\Pi} \frac{\partial F_{\mathrm{rad}}}{\partial z}+\frac{L_{v}}{c_{p} \Pi} \frac{\partial P}{\partial z}
\end{aligned}
$$

which include the effects of the precipitation $P\left(\mathrm{~m} \mathrm{~s}^{-1}\right)$ and net radiative fluxes $F_{\text {rad }}\left(\mathrm{K} \mathrm{m} \mathrm{s}^{-1}\right)$. The overbars have been omitted for notational convenience, except for the turbulent fluxes. Variations of the Exner function throughout the cloud layer are assumed to be negligibly small.

By assuming a well-mixed stratocumulus cloud layer as before, Eqs. (10) can be straightforwardly integrated from cloud base, denoted by a superscript $b$, up to cloud top, which results in 


$$
\begin{aligned}
& h \frac{\partial q_{t}}{\partial t}=w_{e} \Delta q_{t}+{\overline{w^{\prime} q_{t}^{\prime}}}^{b}-\delta P, \\
& h \frac{\partial \theta_{l}}{\partial t}=w_{e} \Delta \theta_{l}+{\overline{w^{\prime} \theta_{l}^{\prime}}}^{b}-\frac{\delta F_{\mathrm{rad}}}{\Pi}+\frac{L_{v}}{c_{p} \Pi} \delta P .
\end{aligned}
$$

The inversion jumps and the entrainment rate $w_{e}$ entered this equation through the use of the flux-jump relation: $\frac{w^{\prime} \varphi^{\prime}}{t}=-w_{e} \Delta \varphi$ (Lilly 1968). Also $\delta$ indicates a difference between cloud top and base, such that for longwave cooling: $\delta F_{\text {rad }}>0$. As the precipitation flux is defined negative downward and no precipitation enters the cloud layer at the top, $\delta P>0$.

When Eqs. (11) are used in Eq. (9), an equation is acquired for the total LWP tendency. This equation can be conveniently split into the contributions of the five relevant processes: turbulent fluxes at cloud base ("Base"), entrainment ("Ent"), radiation ("Rad"), precipitation ("Prec"), and subsidence ("Subs"):

$$
\frac{\partial \mathrm{LWP}}{\partial t}=\mathrm{Ent}+\text { Base }+ \text { Rad }+ \text { Prec }+ \text { Subs },
$$

in which

$$
\begin{aligned}
\text { Ent } & =\rho w_{e}\left(\eta \Delta q_{t}-\Pi \gamma \eta \Delta \theta_{l}-h \Gamma_{q_{l}}\right), \\
\text { Base } & =\rho \eta\left(\overline{w^{\prime} q_{t}^{\prime}} b-\Pi \gamma{\overline{w^{\prime} \theta_{l}^{\prime}}}^{b}\right), \\
\text { Rad } & =\rho \eta \gamma \delta F_{\mathrm{rad}}, \\
\text { Prec } & =-\rho \delta P, \\
\text { Subs } & =-\rho h \Gamma_{q_{l}} \bar{w}\left(z_{i}\right) .
\end{aligned}
$$

Equations (12a)-(12e) allow for the evaluation of the relative contribution of each of the processes to the LWP tendency. Inserting typical values indicates, for instance, that the magnitude of the LWP tendency due to subsidence (about $5 \mathrm{~g} \mathrm{~m}^{-2} \mathrm{~h}^{-1}$ ) is about 9 times as small as that due to radiation. Table 1 gives an overview of the LWP tendencies induced by an increase of $1 \mathrm{~W} \mathrm{~m}^{-2}$ in the cloud-base turbulent and entrainment fluxes, as well as in the precipitation and radiation fluxes.

The entrainment term in Eq. (12a) is typically of similar magnitude as the radiative cooling term. The first two terms between the parentheses in Eq. (12a) represent cloud-thinning tendencies due to entrainment drying and warming. The third term describes cloud thickening due to entrainment. Randall (1984) found that entrainment can result in net cloud thickening despite its cloud-drying and -warming effect. This "cloud deepening through entrainment" occurs only for deep cloud layers (large $h$ ) and/or small inversion jumps $\Delta \theta_{l}$
TABLE 1. Overview of the LWP tendency induced by a $1 \mathrm{~W} \mathrm{~m}^{-2}$ increase in the denoted variables.

\begin{tabular}{lc}
\hline \hline \multicolumn{1}{c}{ Variable } & $\partial \mathrm{LWP} / \partial t\left(\mathrm{~g} \mathrm{~m}^{-2} \mathrm{~h}^{-1}\right)$ \\
\hline$\rho L_{v}{\overline{w^{\prime} q_{t}^{\prime}}}^{b} ; \rho L_{v} w_{e} \Delta q_{t}$ & 0.60 \\
$\rho c_{p} \overline{w^{\prime} \theta_{l}^{\prime}} ; \rho c_{p} w_{e} \Delta \theta_{l} ;-\rho c_{p} \delta F_{\mathrm{rad}} / \Pi$ & -0.82 \\
$\rho L_{v} \delta P$ & -1.44 \\
\hline
\end{tabular}

and $\Delta q_{t}$. He introduced a variable $X$, which is similar to the term between parentheses in Eq. (12a), but only valid for a well-mixed boundary layer, as it assumes that the entrainment drying and warming are spread over the entire depth of the boundary layer.

To assess how the LWP tendency due to entrainment depends on $\kappa$, the definition in Eq. (1) is used to substitute $\Delta q_{\mathrm{t}}$ out of Eq. (12a), which gives

$$
\left.\frac{\partial \mathrm{LWP}}{\partial t}\right|_{\text {Ent }}=\rho \eta w_{e}\left(\frac{c_{p}}{L_{v}} \frac{\Delta \theta_{l}}{\kappa-1}-\Pi \gamma \Delta \theta_{l}-\frac{h \Gamma_{q_{l}}}{\eta}\right) .
$$

It is clear from this equation that the cloud-thinning tendency due to entrainment becomes increasingly large as $\kappa$ increases toward one. The magnitude of the two major cloud-thickening processes-radiative cooling [Eq. (12c)] and input of moisture at cloud base by turbulent fluxes [Eq. (12b)]—are limited by, respectively, the radiative divergence over the cloud layer $\delta F_{\text {rad }} \lesssim$ $60 \mathrm{~W} \mathrm{~m}^{-2}$ and the surface latent heat flux, which is typically between 50 and $150 \mathrm{~W} \mathrm{~m}^{-2}$. The thinning tendency due to entrainment will therefore dominate the LWP tendency for large enough values of $\kappa$, such that the cloud layer will inevitably thin.

\section{Discussion}

\section{a. LWP tendency due to entrainment}

To arrive at a zero-order estimate of the LWP tendency due to entrainment, a qualitative relation for the entrainment rate is used (Stevens et al. 2005):

$$
w_{e}=A \frac{\delta F_{\mathrm{rad}}}{\Delta \theta_{l}}
$$

in which the entrainment efficiency $A$ is of order unity. Typically, microphysical processes, the magnitude of the humidity inversion jump, surface turbulent fluxes, and wind shear over the inversion have important effects on the entrainment efficiency via the buoyancy flux profile (Stevens 2002). The value of $A$ is therefore expected to vary significantly in space and time. Nevertheless, for illustrative purposes, $A$ is treated as a constant. 


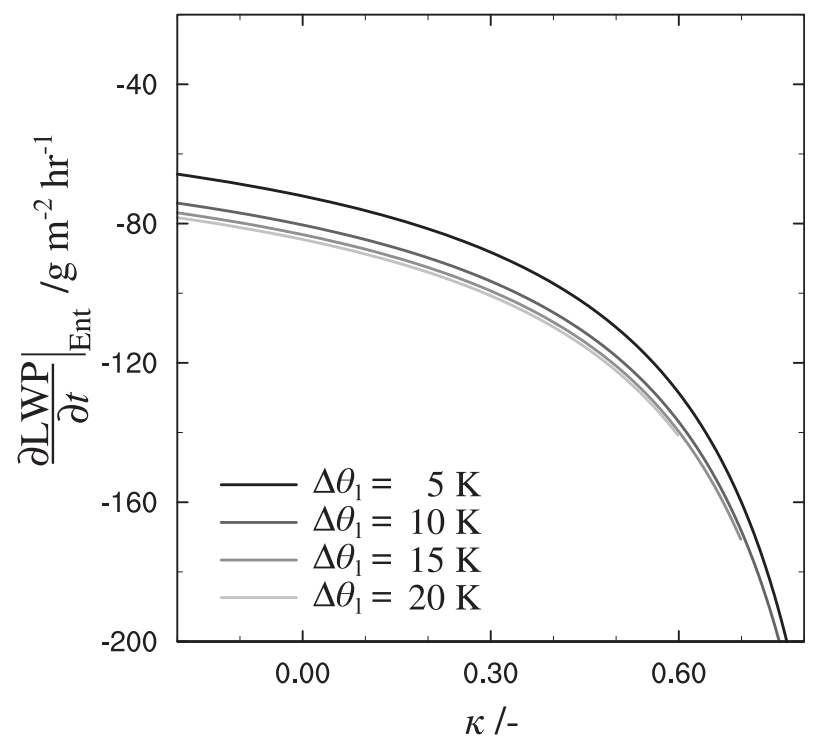

FIG. 2. Contribution of entrainment to the LWP tendency as a function of $\kappa$, as given by Eq. (15) for a set of parameters chosen on the basis of the DYCOMS-II case (Stevens et al. 2005).

Substitution of Eq. (14) into Eq. (13) gives

$$
\left.\frac{\partial \mathrm{LWP}}{\partial t}\right|_{\text {Ent }}=\rho \eta A \delta F_{\mathrm{rad}}\left(\frac{c_{p}}{L_{v}} \frac{1}{\kappa-1}-\Pi \gamma-\frac{h \Gamma_{q_{l}}}{\eta \Delta \theta_{l}}\right) .
$$

The inverse proportionality of the entrainment rate to the $\theta_{l}$ jump over the inversion causes the LWP tendency due to entrainment to be almost independent of $\Delta \theta_{l}$, as the third term on the rhs of Eq. (15) is relatively small compared to the other two.

Figure 2 shows the LWP tendency due to entrainment described by Eq. (15) as a function of $\kappa$ for several values of $\Delta \theta_{l}$. Table 2 shows the parameters that were used for this plot. These parameters were chosen to match as closely as possible the setup of the Second Dynamics and Chemistry of Marine Stratocumulus field study (DYCOMS-II; Stevens et al. 2003a, 2005). This case is particularly interesting, as it lies well within the original buoyancy reversal regime, yet the stratocumulus cloud layer is persistent in the observations (Stevens et al. 2003b). From the figure, it is clear that the cloud-thinning tendency due to entrainment increases rapidly with $\kappa$. For DYCOMS-II, $\kappa \approx 0.55$, such that this thinning tendency exceeds $-120 \mathrm{~g} \mathrm{~m}^{-2} \mathrm{~h}^{-1}$. This indicates that entrainment alone can dissolve the stratocumulus cloud in a matter of hours.

Note that the plot indeed shows that there is hardly any explicit dependence of the cloud thinning tendency on $\Delta \theta_{l}$. Furthermore, the entrainment efficiency and the radiative divergence over the cloud layer are simply
TABLE 2. Overview of the parameters and variables described in the text with the values used. These values are based on the DYCOMS-II case setup (Stevens et al. 2005). Variables $\eta$ and $\Gamma_{q_{l}}$ are derived in the appendix.

\begin{tabular}{ll}
\hline \hline \multicolumn{2}{c}{ Parameters } \\
\hline$p_{s}$ & \\
$T$ & $1017.8 \mathrm{hPa}$ \\
$h$ & $283 \mathrm{~K}$ \\
$z_{i}$ & $200 \mathrm{~m}$ \\
$\rho c_{p} \delta F_{\text {rad }}$ & $800 \mathrm{~m}$ \\
$\rho L_{v} \delta P$ & $48 \mathrm{~W} \mathrm{~m}^{-2}$ \\
$\bar{w}^{\prime} \theta_{l}^{\prime}$ & $0 \mathrm{~W} \mathrm{~m}^{-2}$ \\
$\bar{w}\left(z_{i}\right)$ & $0 \mathrm{~K} \mathrm{~m} \mathrm{~s}^{-1}$ \\
$A$ & $-3.0 \mathrm{~mm} \mathrm{~s}^{-1}$ \\
\hline$\rho$ & 1.3 \\
$q_{s}$ & \\
$\gamma \equiv \frac{\partial q_{s}}{\partial T}=\frac{L_{v} q_{s}}{R_{v} T^{2}}$ & $1.13 \mathrm{~kg} \mathrm{~m}^{-3}$ \\
$\eta \equiv\left(1+\frac{L_{v} \gamma}{c_{p}}\right)^{-1}$ & $8.2 \mathrm{~g} \mathrm{~kg}^{-1}$ \\
$\Gamma_{q_{l}}=g \eta\left(\frac{q_{s}}{R_{d} T}-\frac{\gamma}{c_{p}}\right)$ & $0.55 \mathrm{~g} \mathrm{~kg}^{-1} \mathrm{~K}^{-1}$ \\
\hline
\end{tabular}

coefficients of the tendency in Eq. (12a). Larger values will result in a stronger cloud thinning tendency and will shift the lines in Fig. 2 downward.

So far, only entrainment has been considered. In the next section, source terms for the LWP will also be considered in order to find the conditions for which a stratocumulus cloud layer will thin.

\section{b. LWP source terms}

A stratocumulus cloud layer can be maintained even under conditions with a strong thinning tendency due to entrainment, provided that the opposing cloud thickening processes are strong enough. In terms of Eqs. (12), the total LWP tendency is zero when

$$
\text { Ent }+ \text { Base }+ \text { Rad }+ \text { Prec }+ \text { Subs }=0 .
$$

Straightforward substitution of all terms results in

$$
\begin{gathered}
w_{e}\left(\Delta q_{t}-\Pi \gamma \Delta \theta_{l}\right)+\overline{w^{\prime} q_{t}^{\prime}}-\Pi \gamma \overline{w^{\prime} \theta_{l}^{\prime}} b+\gamma \delta F_{\mathrm{rad}} \\
-\frac{\delta P}{\eta}-\frac{h \Gamma_{q_{l}}}{\eta}\left[w_{e}+\bar{w}\left(z_{i}\right)\right]=0 .
\end{gathered}
$$

This equation can then be easily solved for $\Delta q_{t}$ to give

$$
\begin{aligned}
\Delta q_{t, \mathrm{eq}}= & {\left[\gamma(\Pi A-1) \delta F_{\mathrm{rad}}-{\overline{w^{\prime} q_{t}^{\prime}}}^{b}+\Pi \gamma \overline{w^{\prime} \theta_{l}^{\prime}} b\right.} \\
& \left.+\frac{\delta P}{\eta}+\frac{h \Gamma_{q_{l}}}{\eta} \bar{w}\left(z_{i}\right)\right] \frac{\Delta \theta_{l, \mathrm{eq}}}{A \delta F_{\mathrm{rad}}}+\frac{h \Gamma_{q_{l}}}{\eta},
\end{aligned}
$$




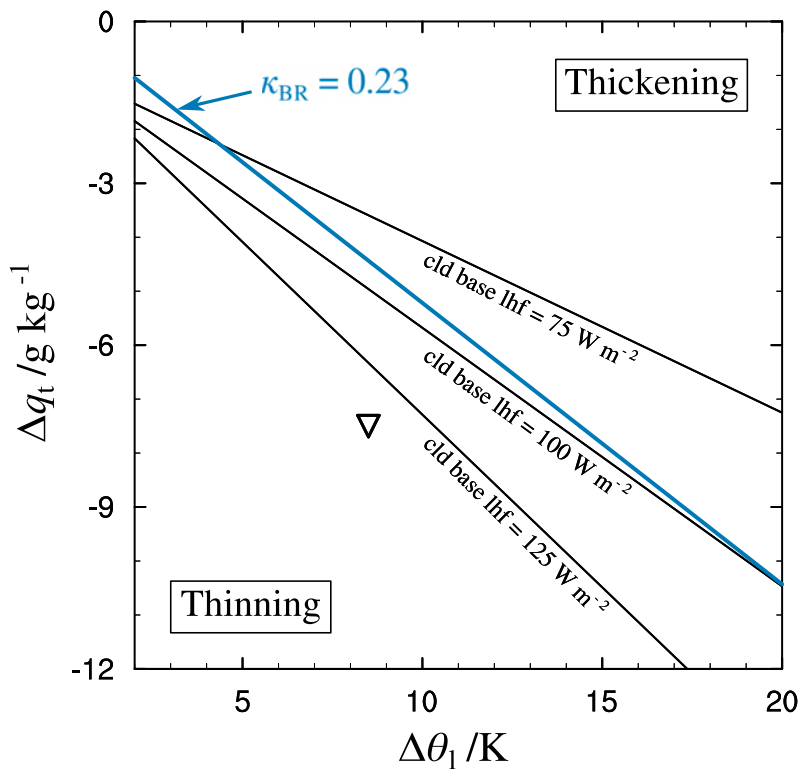

FIG. 3. Plots of the equilibrium lines defined by Eq. (18), for three values of the surface latent heat fluxes: 75,100 , and $125 \mathrm{~W} \mathrm{~m}^{-2}$. The blue line indicates the buoyancy reversal criterion formulated by Randall (1980) and Deardorff (1980), $\kappa_{\mathrm{BR}}=0.23$. The triangle marks the location in phase space of the DYCOMS-II case for which the surface latent heat flux is about $115 \mathrm{~W} \mathrm{~m}^{-2}$.

where the entrainment relation of Eq. (14) has again been substituted. Equation (18) defines a pair of critical inversion jumps $\Delta q_{t, \text { eq }}$ and $\Delta \theta_{l, \text { eq }}$ for which the LWP of the stratocumulus layer is constant in time due to a balance of the sources and sinks. Figure 3 shows these pairs as a function of the latent heat flux at cloud base, again using the parameters in Table 2. Note that the precipitation rate is zero, as it was not included in the original DYCOMS-II simulations. Furthermore, $\overline{w^{\prime} \theta_{l}^{\prime}} b$ is typically small and has therefore been neglected. For the area to the left of the equilibrium lines, the cloud layer is thinning due to additional entrainment drying and warming.

Figure 3 shows that, for a cloud-base latent heat flux of $100 \mathrm{~W} \mathrm{~m}^{-2}$, the equilibrium condition given by Eq. (18) is similar to the buoyancy reversal criterion of Randall (1980) and Deardorff (1980), which is shown in blue. However, Eq. (18) allows for the persistence of stratocumulus clouds beyond the original buoyancy reversal criterion line, depending on the magnitude of the source terms and is therefore in accord with the observations summarized by Kuo and Schubert (1988).

The black triangle marks the location of the DYCOMS-II case in the phase space. The latent heat flux for this case is almost constant with height at about $115 \mathrm{~W} \mathrm{~m}^{-2}$. According to this analysis, the cloud layer is thinning slowly. The rate at which it thins can be calculated using Eqs. (12) and is about $-19 \mathrm{~g} \mathrm{~m}^{-2} \mathrm{~h}^{-1}$. The results shown in Fig. 3, however, are significantly influenced by $A$. For a slightly lower value, $A \approx 1.1$ (corresponding to a reduction of the entrainment velocity of only $1 \mathrm{~mm} \mathrm{~s}^{-1}$ ), the cloud layer would even be thickening. Similarly, a higher cloud-base latent heat flux of about $150 \mathrm{~W} \mathrm{~m}^{-2}$ would provide enough moisture to the cloud layer to keep it from thinning.

In that respect, it is important to note that the analysis presented here is based on the instantaneous state of the cloud layer, which means that interactions among processes are not accounted for. As the LWP of the cloud layer changes as a result of a net tendency, radiative fluxes, precipitation, and entrainment will change accordingly on a relatively short time scale. On a longer time scale, the humidity flux at cloud base and $\Delta q_{t}$ and $\Delta \theta_{l}$ will be affected. The goal of this discussion is therefore not to describe the temporal evolution of a stratocumulus cloud layer, but rather to show how the stratocumulus cloud thinning for sufficiently high values of $\kappa$ can be reasonably expected from mere budget arguments.

To that end, the definition of $\kappa$ in Eq. (1) is now used to substitute out $\Delta q_{T \text {,eq }}$ from Eq. (18). This gives us an equilibrium $\kappa$ value, beyond which the cloud layer is expected to thin. This $\kappa_{\text {eq }}$ can be written as follows:

$$
\begin{aligned}
\kappa_{\mathrm{eq}}= & 1+\frac{{ }_{p} \delta F_{\mathrm{rad}} A}{L_{v}}\left\{\gamma(\Pi A-1) \delta F_{\mathrm{rad}}-\overline{w^{\prime} q_{t}^{\prime}} b\right. \\
& \left.+\Pi \gamma \overline{\bar{w}^{\prime} \theta_{l}^{\prime}} b+\frac{\delta P}{\eta}+\frac{h \Gamma_{q_{l}}}{\eta}\left[\bar{w}\left(z_{i}\right)+\frac{A \delta F_{\mathrm{rad}}}{\Delta \theta_{l}}\right]\right\}^{-1} .
\end{aligned}
$$

In Fig. $4, \kappa_{\mathrm{eq}}$ is shown as a function of $\Delta \theta_{l}$ for three different values of the cloud-base latent heat flux. Clearly, $\kappa_{\mathrm{eq}}$ is higher for larger cloud-base latent heat fluxes, while it is only a weak function of $\Delta \theta_{l}$. This is in good agreement with the results of the LES experiments performed by Xiao et al. (2011), who found that for well-mixed boundary layers and hence a maximum moisture supply from the surface, the cloud layer breaks up at larger values of $\kappa$ as compared to decoupled cases. It is also in qualitative agreement with the LES results of the stratocumulus transitions presented in Fig. 1. The stratocumulus cloud layer in the ASTEX case breaks up for significantly smaller $\kappa$ values than in the composite cases. The LWP budget analysis shows that this can be reasonably expected on the basis of the surface latent heat flux, which is on average about a factor of 2 smaller for ASTEX.

\section{c. The Klein and Hartmann line}

From observations, Klein and Hartmann (1993) found a strong correlation between the seasonally averaged 


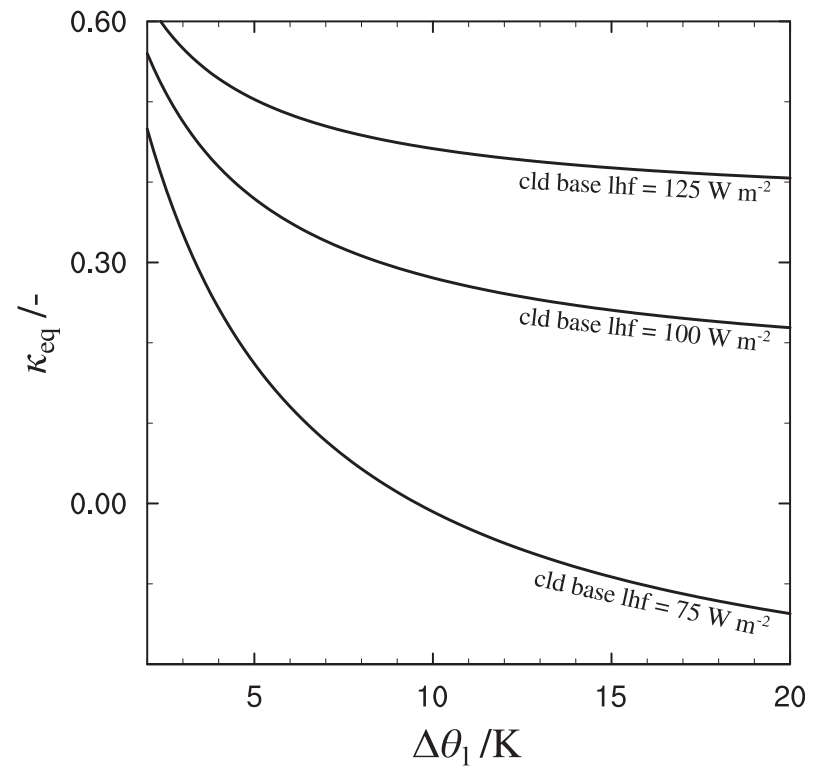

FIG. 4. The equilibrium inversion stability parameter, given by Eq. (19), for three values of the latent heat flux at cloud base: 75, 100 , and $125 \mathrm{~W} \mathrm{~m}^{-2}$.

lower-tropospheric stability (LTS) and cloud cover in the main subtropical stratocumulus regions. The linear fit through the data points has become known as the Klein and Hartmann line and is described by

$$
\sigma=a \mathrm{LTS}+b,
$$

in which $\sigma$ is the cloud cover and $a=5.7 \mathrm{~K}^{-1}$ and $b=$ -55.73 are fitting constants. Chung et al. (2012) later qualitatively derived this relationship from a steadystate, large-scale $\theta_{l}$ budget. Here, we express Eq. (20) as a function of $\kappa$ in order to show some analogy with the results derived from LESs by Moeng (2000) and Lock (2009).

Assuming a constant $\theta$ lapse rate in the free atmosphere $\Gamma_{\theta}$, the LTS can be related to the inversion jump of $\theta_{l}$ as follows:

$$
\mathrm{LTS}=\Delta \theta_{l}-\left(z_{700}-z_{i}\right) \Gamma_{\theta} .
$$

Here, $z_{700} \approx 3000 \mathrm{~m}$ is the height of the $700-\mathrm{hPa}$ isobar. Substitution of Eqs. (1) and (21) into Eq. (20) results in the following relation between $\sigma$ and $\kappa$ :

$$
\sigma=\frac{a L_{v}}{c_{p}}(\kappa-1) \Delta q_{t}-a \Gamma_{\theta}\left(z_{700}-z_{i}\right)+b .
$$

Figure 5 shows $\sigma$ as a function of $\kappa$, using $\Gamma_{\theta}=-6 \mathrm{~K} \mathrm{~km}^{-1}$ and $z_{i}=800 \mathrm{~m}$. This figure shows that the seasonally averaged cloud cover decreases linearly with $\kappa$. Interestingly,

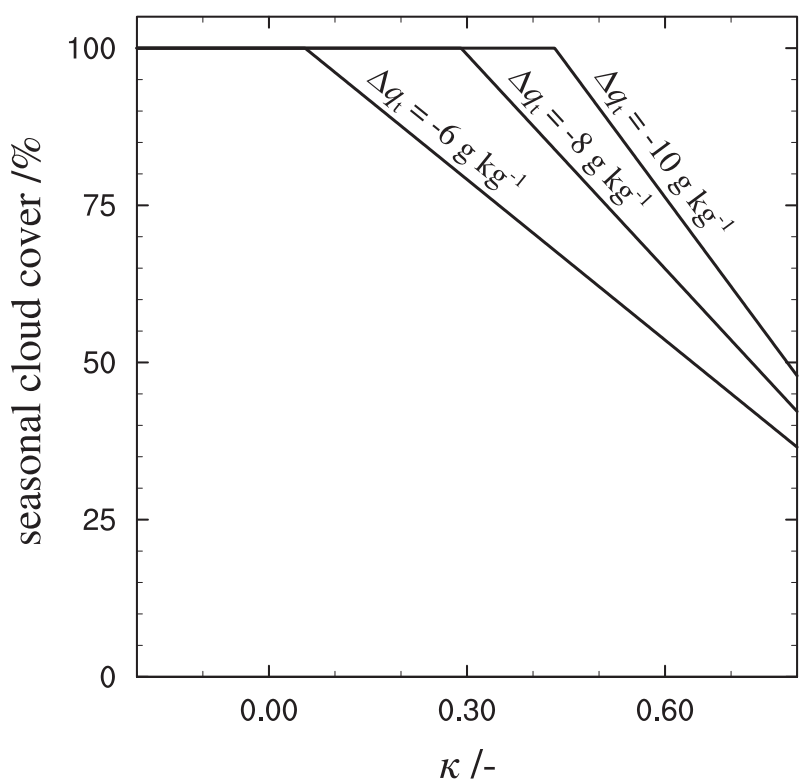

FIG. 5. Seasonal cloud cover as a function of $\kappa$ based on the Klein and Hartmann line as described by Eq. (22), using $z_{i}=800 \mathrm{~m}$ and $\Gamma_{\theta}=-6 \mathrm{~K} \mathrm{~km}^{-1}$. Function is capped at $\sigma=100 \%$.

the cloud cover is qualitatively similar to those found from the LES process studies depicted in Fig. 1 and by Lock (2009), despite the fact that the Klein and Hartmann line describes a fit through large-area and seasonally averaged observations. However, whereas the Klein and Hartmann line describes the cloud cover as a function of the LTS only, the $\kappa$ analysis suggests that additionally $\Delta q_{t}$ and the latent heat flux should be taken into account. An analysis of the correlation between the relative humidity in the free atmosphere and the cloud fraction from observations would therefore be very insightful.

\section{Conclusions}

In this article it is argued that the breakup of the subtropical marine stratocumulus clouds for high values of the inversion stability parameter $\kappa$ can be satisfactorily explained using simple cloud-layer budget arguments.

A budget equation was derived for the LWP of an adiabatic stratocumulus cloud layer, such that the contributions of the different physical mechanisms could be separately analyzed. Using a phenomenological entrainment relation, it is shown that the cloud-thinning tendency due to entrainment increases rapidly with $\kappa$, making cloud breakup inevitable for sufficiently large values of $\kappa$.

The conditions for which the cloud layer is neither thickening nor thinning could be found using the LWP tendency equation. This allowed us to define 

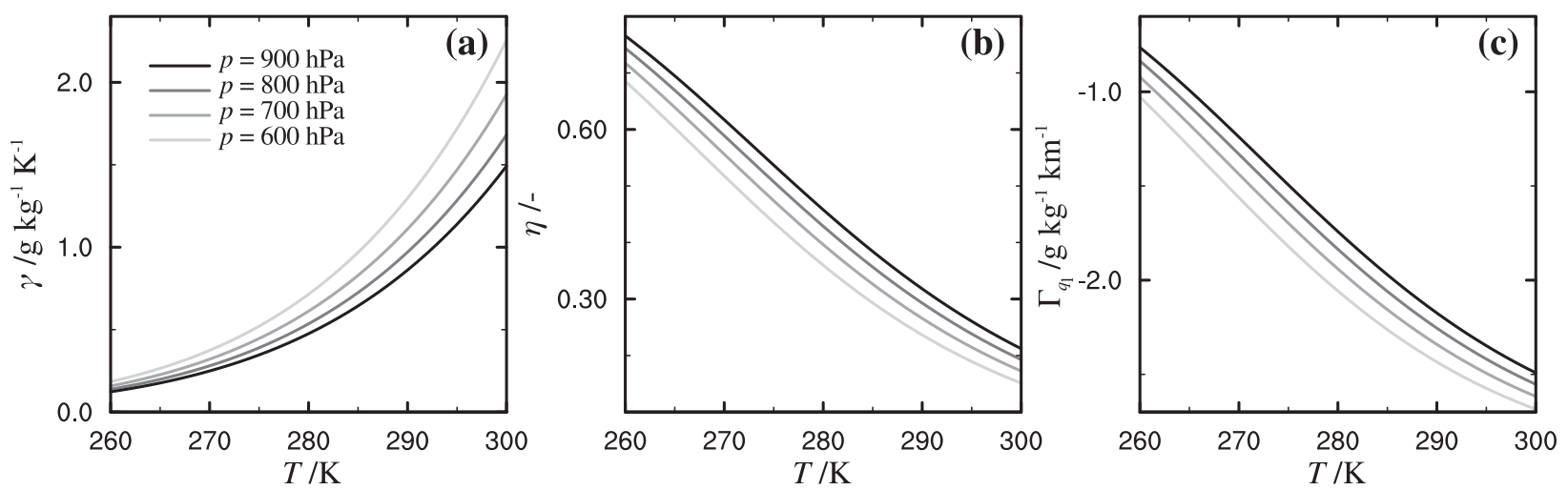

FIG. A1. (a) Clausius-Clapeyron relation $\gamma$, (b) $\eta$, and (c) $\Gamma_{q_{l}}$ as a function of temperature for the pressures specified in the legend.

an equilibrium value of $\kappa$ beyond which the cloud layer will thin. The value of $\kappa_{\text {eq }}$ is mainly determined by the turbulence humidity flux at cloud base and the entrainment efficiency parameter $A$. The results are in qualitative agreement with the findings of Xiao et al. (2011), who show that the $\kappa$ value for which clouds start to break up are lower for decoupled than for well-mixed boundary layers.

Finally, it was shown that the linear relationship between the LTS and the cloud cover, found from observations by Klein and Hartmann (1993), also describes a cloud cover that decreases with $\kappa$.

Acknowledgments. The investigations were done as part of the European Union Cloud Intercomparison, Process Study and Evaluation (EUCLIPSE) project, funded under the Seventh Framework Programme of the European Union. The work was sponsored by the National Computing Facilities Foundation (NCF) for the use of supercomputer facilities. We kindly thank Roel Neggers for the stimulating discussions, as well as Steef Böing and three anonymous reviewers for their helpful comments on the manuscript.

\section{APPENDIX}

\section{Partial Derivatives of the Liquid Water Specific Humidity}

Equation (2) contains partial derivatives of $q_{l}$ with respect to $q_{t}, \theta_{l}$, and $z_{i}$. Below, expressions for each of these derivatives are derived.

\section{a. Total humidity}

First, the definition of $\theta_{l}$ is written in an incremental form:

$$
d \theta_{l}=d \theta-\frac{L_{v}}{\Pi c_{p}} d q_{l}
$$

Now, because $\theta_{l}$ is kept constant, the lhs of Eq. (A1) is zero, such that

$$
d T=\frac{L_{v}}{c_{p}} d q_{l}
$$

In a saturated environment, $q_{t}$ can be written as the sum of the saturation specific humidity $q_{s}$ and $q_{l}$, or, in its incremental form:

$$
d q_{t}=d q_{s}+d q_{l}
$$

Equation (A2) can now be used to substitute $d q_{l}$ out of this equation, giving

$$
d q_{t}=d q_{s}+\frac{c_{p}}{L_{v}} d T=\left(1+\frac{c_{p}}{L_{v} \gamma}\right) d q_{s}
$$

where $\gamma$ is defined as

$$
\gamma \equiv \frac{\partial q_{s}}{\partial T}=\frac{L_{v} q_{s}}{R_{v} T^{2}}
$$

The second equality in Eq. (A5) is given by the ClausiusClapeyron relation. Figure A1a shows $\gamma$ for a range of relevant temperatures and pressures. Using this expression to eliminate $d q_{s}$ in Eq. (A3) and rearranging the terms gives

$$
d q_{t}=\left(\frac{L_{v} \gamma}{c_{p}+L_{v} \gamma}\right) d q_{t}+d q_{l}
$$

which can be solved for $d q_{t}$. The partial derivative can then be written as

$$
\frac{\partial q_{l}}{\partial q_{t}}=\eta
$$

in which 


$$
\eta \equiv\left(1+\frac{L_{v} \gamma}{c_{p}}\right)^{-1}
$$

This $\eta$ can be interpreted as an efficiency at which increments in $q_{t}$ are converted to $q_{l}$. Figure A1b shows $\eta$ as a function of temperature for different pressures.

\section{b. Liquid water potential temperature}

For increments of $\theta$, the following can be written:

$$
d \theta=\frac{d T}{\Pi}=\frac{1}{\Pi} \frac{\partial T}{\partial q_{s}} d q_{s} .
$$

Furthermore, in a saturated environment at constant $q_{t}$,

$$
d q_{s}=-d q_{l} .
$$

Equations (A9) and (A10) can be used in Eq. (A1) to give

$$
d \theta_{l}=-\frac{1}{\Pi}\left(\frac{1}{\gamma}+\frac{L_{v}}{c_{p}}\right) d q_{l} .
$$

The partial derivative of $q_{l}$ with respect to $\theta_{l}$ can then be written as follows:

$$
\frac{\partial q_{l}}{\partial \theta_{l}}=-\Pi \gamma \eta,
$$

with $\eta$ as defined by Eq. (A8).

\section{c. Inversion height}

In the cloud layer, the following can be written for the lapse rate of the liquid water specific humidity $\Gamma_{q_{l}}$ :

$$
\Gamma_{q_{l}}=-\frac{\partial q_{l}}{\partial z}=\frac{\partial q_{s}}{\partial z} .
$$

Since $q_{s}=f(T, p)$, partial differentiation can be used to write

$$
\Gamma_{q_{l}}=\frac{\partial q_{s}}{\partial z}=\frac{\partial q_{s}}{\partial T} \frac{\partial T}{\partial z}+\frac{\partial q_{s}}{\partial p} \frac{\partial p}{\partial z} .
$$

Rewriting the first term on the right-hand side gives

$$
\frac{\partial q_{s}}{\partial T} \frac{\partial T}{\partial z}=-\gamma \Gamma_{T}
$$

where $\Gamma_{T}$ is the lapse rate of temperature. Since Eq. (A14) should be evaluated in cloud, $\Gamma_{T}$ is equal to the saturated adiabatic lapse rate:

$$
\Gamma_{T, s} \approx \frac{g}{c_{p}}+\frac{L_{v}}{c_{p}} \frac{\partial q_{s}}{\partial z},
$$

in which $q_{v}$ in the partial derivative has been replaced by $q_{s}$.

To rewrite the second term on the right-hand side, $q_{s}$ is approximated as follows (e.g., Stull 1988):

$$
q_{\mathrm{s}} \approx \frac{\varepsilon e_{\mathrm{s}}}{p} .
$$

Here, $e_{s}$ is the saturation pressure and $\varepsilon=R_{d} / R_{v} \approx 0.622$ is the ratio of the gas constant of dry air to that of water vapor. Differentiating this equation with respect to $p$ results in

$$
\frac{\partial q_{s}}{\partial p}=-\frac{q_{s}}{p} .
$$

The atmosphere is furthermore assumed to be in hydrostatic equilibrium,

$$
\frac{\partial p}{\partial z}=-\rho g \approx-\frac{p g}{R_{d} T} .
$$

Substitution of Eqs. (A15)-(A19) into Eq. (A14) gives

$$
\left(1+\frac{\gamma L_{v}}{c_{p}}\right) \frac{\partial q_{s}}{\partial z}=-\frac{g \gamma}{c_{p}}+\frac{g q_{s}}{R_{d} T},
$$

such that after rewriting

$$
\Gamma_{q_{l}}=g \eta\left(\frac{q_{s}}{R_{d} T}-\frac{\gamma}{c_{p}}\right) .
$$

The lapse rate is approximately $-1.8 \mathrm{~g} \mathrm{~kg}^{-1} \mathrm{~km}^{-1}$ in the relevant temperature and pressure range (see Fig. A1c). As a result of mainly precipitation, stratocumulus clouds are typically subadiabatic (Wood 2005). Equation (A21) therefore gives an upper limit for magnitude of the lapse rate, which can in practice be up to $40 \%$ smaller.

\section{REFERENCES}

Albrecht, B. A., C. S. Bretherton, D. Johnson, W. H. Scubert, and A. S. Frisch, 1995: The Atlantic Stratocumulus Transition Experiment-ASTEX. Bull. Amer. Meteor. Soc., 76, 889-904.

Bretherton, C. S., P. N. Blossey, and C. R. Jones, 2013: Mechanisms of marine low cloud sensitivity to idealized climate perturbations: A single-LES exploration extending the CGILS cases. J. Adv. Model. Earth Syst., 5, 316-337, doi:10.1002/ jame.20019.

Chung, D., G. Matheou, and J. Teixeira, 2012: Steady-state largeeddy simulations to study the stratocumulus to shallow cumulus cloud transition. J. Atmos. Sci., 69, 3264-3276. 
Deardorff, J. W., 1980: Cloud top entrainment instability. J. Atmos. Sci., 37, 131-147.

Duynkerke, P. G., 1993: The stability of cloud top with regard to entrainment: Amendment of the theory of cloud-top entrainment instability. J. Atmos. Sci., 50, 495-502.

Heus, T., and Coauthors, 2010: Formulation of the Dutch Atmospheric Large-Eddy Simulation (DALES) and overview of its applications. Geosci. Model Dev., 3, 415-444, doi:10.5194/ gmd-3-415-2010.

Klein, S. A., and D. L. Hartmann, 1993: The seasonal cycle of low stratiform clouds. J. Climate, 6, 1587-1606.

Kuo, H.-C., and W. H. Schubert, 1988: Stability of cloud-topped boundary layers. Quart. J. Roy. Meteor. Soc., 114, 887-916, doi:10.1002/qj.49711448204.

Lilly, D. K., 1968: Models of cloud-topped mixed layers under a strong inversion. Quart. J. Roy. Meteor. Soc., 94, 292-309, doi:10.1002/qj.49709440106.

Lock, A. P., 2009: Factors influencing cloud area at the capping inversion for shallow cumulus clouds. Quart. J. Roy. Meteor. Soc., 135, 941-952, doi:10.1002/qj.424.

MacVean, M. K., and P. J. Mason, 1990: Cloud-top entrainment instability through small-scale mixing and its parameterization in numerical models. J. Atmos. Sci., 47, 1012-1030.

Mellado, J. P., B. Stevens, H. Schmidt, and N. Peters, 2009: Buoyancy reversal in cloud-top mixing layers. Quart. J. Roy. Meteor. Soc., 135, 963-978, doi:10.1002/qj.417.

Moeng, C.-H., 2000: Entrainment rate, cloud fraction, and liquid water path of PBL stratocumulus clouds. J. Atmos. Sci., 57, 3627-3643.

Noda, A. T., K. Nakamura, T. Iwasaki, and M. Satoh, 2013: A numerical study of a stratocumulus-topped boundary-layer: Relations of decaying clouds with a stability parameter across inversion. J. Meteor. Soc. Japan, in press.

Park, S., C. B. Leovy, and M. A. Rozendaal, 2004: A new heuristic Lagrangian marine boundary layer cloud model. J. Atmos. Sci., 61, 3002-3024.

Randall, D. A., 1980: Conditional instability of the first kind upsidedown. J. Atmos. Sci., 37, 125-130.

- 1984: Stratocumulus cloud deepening through entrainment. Tellus, 36A, 446-457, doi:10.1111/j.1600-0870.1984.tb00261.x.

Sandu, I., and B. Stevens, 2011: On the factors modulating the stratocumulus to cumulus transitions. J. Atmos. Sci., 68, 1865-1881.
Siems, S. T., C. S. Bretherton, M. B. Baker, S. Shy, and R. E. Breidenthal, 1990: Buoyancy reversal and cloud-top entrainment instability. Quart. J. Roy. Meteor. Soc., 116, 705-739, doi:10.1002/qj.49711649309.

Stephens, G. L., 2005: Cloud feedbacks in the climate system: A critical review. J. Climate, 18, 237-273.

Stevens, B., 2002: Entrainment in stratocumulus-topped mixed layers. Quart. J. Roy. Meteor. Soc., 128, 2663-2690, doi:10.1256/ qj.01.202.

— , and Coauthors, 2003a: Dynamics and Chemistry of Marine Stratocumulus-DYCOMS-II. Bull. Amer. Meteor. Soc., 84, 579-593.

— marine stratocumulus. Quart. J. Roy. Meteor. Soc., 129, 34693493, doi:10.1256/qj.02.202.

— , and Coauthors, 2005: Evaluation of large-eddy simulations via observations of nocturnal marine stratocumulus. Mon Wea. Rev., 133, 1443-1462.

Stull, R., 1988: An Introduction to Boundary Layer Meteorology. Kluwer Academic, 666 pp.

Van der Dussen, J. J., and Coauthors, 2013: The GASS/EUCLIPSE model intercomparison of the stratocumulus transition as observed during ASTEX: LES results. J. Adv. Model. Earth Syst., 5, 483-499, doi:10.1002/jame.20033.

Wang, Q., and D. H. Lenschow, 1995: An observational study of the role of penetrating cumulus in a marine stratocumulustopped boundary layer. J. Atmos. Sci., 52, 2778-2787.

Wood, R., 2005: Drizzle in stratiform boundary layer clouds. Part I: Vertical and horizontal structure. J. Atmos. Sci., 62, 30113033.

__ , and C. S. Bretherton, 2004: Boundary layer depth, entrainment, and decoupling in the cloud-capped subtropical and tropical marine boundary layer. J. Climate, 17, 35763588.

Xiao, H., C.-M. Wu, and C. R. Mechoso, 2011: Buoyancy reversal, decoupling and the transition from stratocumulus to shallow cumulus topped marine boundary layers. Climate Dyn., 37, 971-984, doi:10.1007/s00382-010-0882-3.

Yamaguchi, T., and D. A. Randall, 2008: Large-eddy simulation of evaporatively driven entrainment in cloud-topped mixed layers. J. Atmos. Sci., 65, 1481-1504. 